\title{
Development of a Non-Motor Fluctuation Assessment Instrument for Parkinson Disease
}

\author{
Galit Kleiner-Fisman, ${ }^{1}$ Rebecca Martine, ${ }^{2}$ Anthony E. Lang, ${ }^{3}$ and Matthew B. Stern ${ }^{2,4}$ \\ ${ }^{1}$ Baycrest Hospital, University of Toronto, 3560 Bathurst Street, Toronto, ON, Canada M6A 2E1 \\ ${ }^{2}$ Parkinson's Disease Research Education and Clinical Center (PADRECC), Philadelphia VA Medical Center, \\ Philadelphia, PA 19107, USA \\ ${ }^{3}$ Toronto Western Hospital, University of Toronto, Toronto, ON, Canada M5I 2 S8 \\ ${ }^{4}$ School of Medicine, University of Pennsylvania, Philadelphia, PA 19104, USA
}

Correspondence should be addressed to Galit Kleiner-Fisman, gkleinerfisman@yahoo.com

Received 25 April 2011; Accepted 24 May 2011

Academic Editor: Alan R. Crossman

Copyright ( 2011 Galit Kleiner-Fisman et al. This is an open access article distributed under the Creative Commons Attribution License, which permits unrestricted use, distribution, and reproduction in any medium, provided the original work is properly cited.

Patients with Parkinson disease are increasingly recognized to suffer from non-motor symptoms in addition to motor symptoms. Many non-motor symptoms fluctuate in parallel with motor symptoms and in relationship to plasma levodopa levels. Though these symptoms are troublesome and result in reduced quality of life to patients and their caregivers, there has not been an objective method of recognizing and quantifying non-motor fluctuations (NMFs). This study sought to develop a patient-based instrument that would accurately capture the experience of patients with NMFs. Patient-based nominal group technique sessions, focus groups, and expert opinion were utilized in developing this questionnaire.

\section{Introduction}

Non-motor symptoms in Parkinson Disease (PD) are increasingly recognized as a major source of disability for patients with moderate to advanced PD. Disability due to these symptoms arises as a result of problems with, among other difficulties, sleep, cognitive and mood disturbances, pain and other sensory complaints, as well as bowel and bladder dysfunction $[1,2]$. Many are poorly responsive to dopaminergic drug replacement given their partial mediation through other (nondopaminergic) neurotransmitter systems [3].

Non-motor fluctuations (NMFs), in contrast, are nonmotor symptoms that vary according to plasma dopaminergic tone in a manner similar to motor fluctuations [4]. While it is likely that these NMFs are highly amenable to medical (dopamine replacement) and surgical interventions, there has been no instrument available to assess presence of NMFs, and this has limited the assessment of symptomatic burden and, therefore, efforts to pursue interventions. Even if such interventions were readily available, however, the lack of a reliable and valid instrument to assess the presence of NMFs would limit measurement of efficacy in clinical trials. As such, the objective of this study was to develop an instrument that assesses the presence of NMFs in individuals with PD and does so with reliability and validity.

\section{Methods}

2.1. Participants. As individuals with motor fluctuations are thought to be at greater risk for NMFs, individuals with motor fluctuations were recruited by (i) mailing pamphlets about the study and its eligibility criteria to all the Parkinson's Disease Research, Education \& Clinical Center (PADRECC) patients at the Philadelphia VA Medical Center (PVAMC), (ii) distributing similar pamphlets at PD patient and caregiver support groups, and at the Pennsylvania Hospital Movement Disorders Center reception area, and by (iii) direct questioning of patients during a routine health care visit if they were listed as having symptoms of NMFs in the PADRECC electronic database. A screening questionnaire was administered to those with motor fluctuations who 


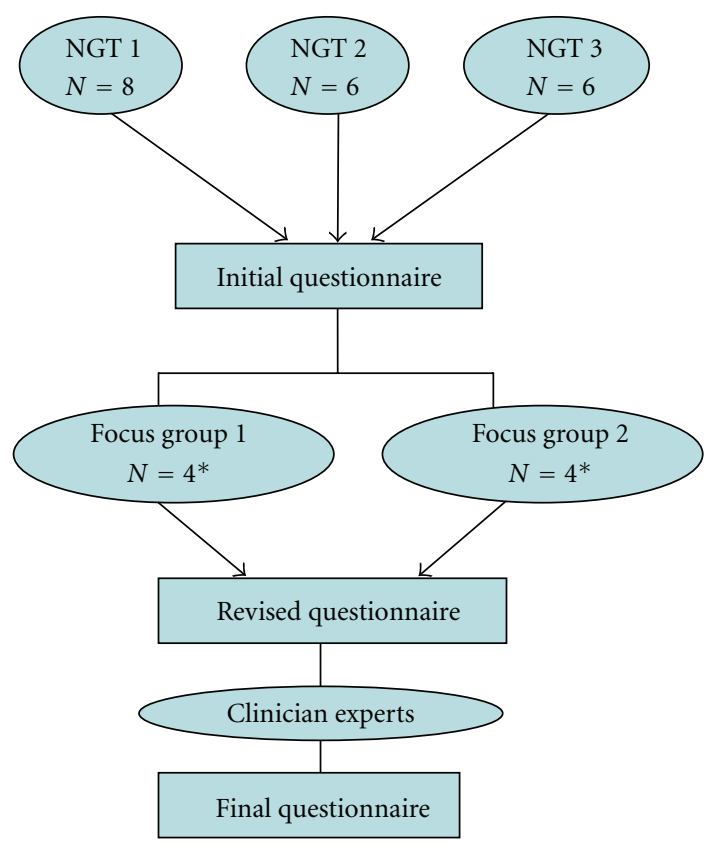

FIGURE 1: NMF questionnaire development schema. NGT: nominal group technique. ${ }^{*}$ Focus group consisted of both new subjects and original members of the NGT sessions.

expressed interest in study participation to verify the presence of motor fluctuations and to assess level of awareness and knowledge about non-motor symptoms. Anyone with substantial cognitive impairment, defined as a Mini-Mental Status Exam (MMSE) score $\leq 24$ (whereby scores had been obtained by any health care provider in the previous six months), was excluded [5].

The study was approved by the Institutional Review Boards (IRB) of the PVAMC and the Pennsylvania Hospital. All participants signed the IRB-approved, written informed consent form before participation.

\subsection{Group Discussion Using Nominal Group Technique.} Three group discussions were held to generate content for the questionnaire. Three to four unique patient and partner/ caregiver dyads were recruited for each session (Figure 1). Nominal Group Technique (NGT) was employed [6]. Each NGT group began with the group facilitator (G.K-F.) reading from a semistructured interview script that operationally defined NMFs (symptoms related to PD that affect functions of the body other than movements, that come and go throughout the day depending on medication response).

Participants were asked to identify and record on paper all NMFs affecting them with particular attention paid to those NMFs that affected day-to-day life and/or life quality. Participants then took turns presenting their written responses such that with each cycle around the table the participant offered a single response. Every response offered by a group member was discussed by the whole group until all participants understood the NMF symptom offered and reached consensus on the language best describing that symptom. Precipitating and aggravating factors for each NMF symptom was discussed as was whether it was
TABLE 1: Characteristics of patient-participants.

\begin{tabular}{lc}
\hline Characteristics & Patients $(n=11)$ \\
\hline Mean age, years & 74 \\
Mean age onset of PD, years & 64 \\
Mean PD stage $(\mathrm{H}+\mathrm{Y})$, years & 3 \\
Mean LEDD, mg/d & 770 \\
Sex, \% men & 91 \\
\hline
\end{tabular}

correlated with a particular motor state (e.g., ON versus OFF).

"Round-robins" continued until all unique responses were exhausted, after which a (secret ballot) vote took place to determine which symptoms were most frequent and disabling. A final composite list for each session was compiled before the group discussion ended and the group reviewed the findings. Group discussions not only identified content for instrument construction using lay language, they also applied meaning to the content and provided a sense of its relative perceived importance.

The complete list of all candidate symptoms identified by patient and caregivers were subjected to further critique by clinicians with expertise in PD. The clinicians were selected from the panel on non-motor symptoms of PD of the Quality Standards Subcommittee of the American Academy of Neurology [7]. Critique focused on frequency of and disability incurred by each symptom with the intention of reducing the number of total items to limit the burden of completing a lengthy questionnaire. Clinician experts' critique led to creation of an initial questionnaire. In constructing this initial questionnaire, a 7 th grade reading level was sought.

2.3. Focus Groups. The initial questionnaire was presented to two focus groups that consisted both of previous participants from NGT sessions and new patients. Participants were asked to critique the questionnaire for item relevance and ease of understanding, and whether response choices were both exhaustive and mutually exclusive. Important gaps in content were identified along with suggestions for questions that should be included to capture the content. Unnecessary or duplicative questions also were identified and removed. Following this process, a revised questionnaire was distributed to clinician experts for a final review.

2.4. Final Questionnaire. An additional goal of the final questionnaire was to create response options that would allow differentiation between non-motor symptoms that were present but did not fluctuate according to plasma dopaminergic tone, and those that did fluctuate. A scoring scheme was developed that consisted of imputing one to three points (mild, moderate, severe) for each endorsed item that indicated a symptom that fluctuated by "ON" versus "OFF" status, and 0 for all other options. As such, higher scores would reflect a greater number and severity of NMFs. A total NMF score ranges from 0 to 84 , and subscores $(\mathrm{mood} / \mathrm{cognition}$, autonomic, sensory, sleep, and fatigue) could be generated in the "ON" and "OFF" periods. 
TABLE 2: Ranking of non-motor fluctuations (NMFs) symptoms by study participants* .

\begin{tabular}{|c|c|c|c|c|}
\hline Rank & Symptom & Frequency & Importance score* & Summary score ${ }^{\dagger}$ \\
\hline 1 & Pain (OFF) & 10 & 50 & 500 \\
\hline 2 & Confusion (OFF) & 9 & 42 & 378 \\
\hline 3 & Poor concentration (OFF) & 9 & 41 & 369 \\
\hline 4 & Frustration (OFF) & 8 & 40 & 320 \\
\hline 5 & Urinary frequency $(\mathrm{ON})$; urgency/incontinence $(\mathrm{OFF})$ & 7 & 33 & 231 \\
\hline 6 & Word-finding difficulty (OFF) & 7 & 33 & 231 \\
\hline 7 & Word-finding difficulty (OFF) & 7 & 25 & 175 \\
\hline 8 & Drooling (OFF) & 6 & 23 & 138 \\
\hline 9 & Poor short-term memory (OFF) & 5 & 25 & 125 \\
\hline 10 & Obsessive/compulsive behavior (OFF) & 6 & 20 & 120 \\
\hline 11 & Poor judgment (OFF) & 5 & 15 & 75 \\
\hline 12 & Depression (OFF) & 5 & 14 & 70 \\
\hline 13 & Mood swings/emotional lability/irritability (OFF) & 5 & 12 & 60 \\
\hline 14 & Insomnia (ON, OFF) & 3 & 14 & 42 \\
\hline 15 & Agitation/irritability/impatience (OFF) & 3 & 10 & 30 \\
\hline 16 & Lack of interest (OFF) & 3 & 9 & 27 \\
\hline 17 & Decreased laughter (apathy?) (OFF) & 2 & 12 & 24 \\
\hline 18 & Difficulty breathing (OFF) & 2 & 11 & 22 \\
\hline 19 & Fatigue (OFF) & 3 & 7 & 21 \\
\hline 20 & Hallucinations (OFF) & 2 & 10 & 20 \\
\hline 21 & Increased perspiration/odor (ON) & 2 & 7 & 14 \\
\hline 22 & Paranoia (OFF) & 2 & 6 & 12 \\
\hline 23 & Double/blurry vision (OFF) & 2 & 5 & 10 \\
\hline 24 & Decreased communication/social withdrawal (OFF) & 1 & 7 & 7 \\
\hline 25 & Decreased reading comprehension (OFF) & 1 & 6 & 6 \\
\hline 26 & Reduced coping skills (OFF) & 1 & 6 & 6 \\
\hline 27 & Reduced sense of taste (OFF) & 1 & 5 & 5 \\
\hline 28 & Tingling (OFF) & 1 & 5 & 5 \\
\hline 29 & Constipation (ON) & 1 & 5 & 5 \\
\hline 30 & Change in hearing (OFF) & 1 & 3 & 3 \\
\hline 31 & Intermittently sleepy (OFF) & 1 & 3 & 3 \\
\hline 32 & Numbness $(\mathrm{OFF})^{\ddagger}$ & 0 & 0 & 0 \\
\hline 33 & Restlessness (ON) & 0 & 0 & 0 \\
\hline
\end{tabular}

${ }^{*}$ Summation of individual scores.

${ }^{\dagger}$ Summary score: frequency X score.

${ }^{\ddagger}$ Mentioned as symptom but not ranked in top 7 .

"Frequency: number of subjects ranking symptom.

19 participants asked to rank symptoms from 1-7 (least to most important).

133 total possible.

121/133-some subjects did not rank all 7.

\section{Results}

3.1. Patients. Baseline characteristics of patient-participants are detailed in Table 1. A total of 11 patients and 11 caregivers participated in NGT and focus groups. All patient participants had motor and non-motor fluctuations. Patientparticipants were male with the exception of one woman (the majority of patient-participants were recruited from the male-predominant VA-based PADRECC).

3.2. Initial Questionnaire. Table 2 is a list of 33 symptoms that was constructed using all symptoms reported during the three sessions. These were then ranked from highest to lowest according to a "summary score" that was calculated by multiplying the number of participants who had reported the symptom by the mean rank of importance that had been reported by participants for that symptom. Symptoms reported by participants but not ranked as important were included in the table.

Clinician experts collapsed some items they believed to be assessing the same underlying concept into one single item (e.g., decreased reading comprehension and poor concentration and decreased communication and word-finding difficulties) and removed other items they thought were not 
highly prevalent in clinical practice (e.g., altered hearing). In those situations where multiple same-construct symptoms were collapsed into a single item, wording was revised to capture the concept appropriately.

The final revised questionnaire resulting from focus groups with patient/caregiver/ and feedback from clinician experts is shown in the appendix. Twenty-eight items were included in the final questionnaire.

\section{Discussion}

Outcome assessments based on patient perceptions and selfreports are increasingly incorporated into clinical trials of patients with PD. No instrument existed previously to allow assessment of NMFs in patients with PD. This study has led to the creation of such an instrument, the Non-motor Fluctuations Assessment instrument (NoMoFA), which can be used as a patient-based outcome measure in both research and clinical practice. While this instrument was developed using methods that impart substantial face and construct validity, reliability and additional validity assessments of the instrument necessarily must follow. To that end, a recent effort to identify wearing off phenomena both motor and non-motor, determined through expert consensus and literature review, identified similar symptoms to our patientderived items [8].

Though patients have long complained of non-motor symptoms to their health-care providers, only recently have they been recognized as important and disabling [9]. Even amongst Movement Disorder specialists, attention to these problems has been limited [10]. Unfortunately, there is a discrepancy between the prevalence of NMFs and the limited degree to which these symptoms are attended to by health care professionals. However, studies indicate that NMFs are common and contribute significantly to reduction in quality of life. As shown by Witjas et al., up to one-third of patients reported greater disability from NMFs than from motor symptoms [9].

Due to the recent increased attention given to NMFs, a new effort has sought to incorporate evaluation of nonmotor symptoms into the standardized PD evaluation protocol $[8,11,12]$. The questionnaire reported here will increase the likelihood that such a standardized evaluation will occur and will do so using an instrument with patient-derived content and with use of vocabulary obtained from patients themselves.

\section{Conclusion}

In developing the NoMoFA, we sampled a heterogeneous group of patients with diverse backgrounds. In addition, we received feedback from experts providing revisions with an effort to maximize clinical relevance. We believe this has increased the likelihood the NoMoFA is an accurate, understandable, comprehensive compilation of NMFs experienced by PD patients. Further work needs to be performed to ensure that the NoMoFA is reliable and valid before it can be incorporated into standard research and clinical evaluations of patients.

\section{Appendix}

\section{Non-Motor Fluctuation Assessment Instrument (NoMoFA)}

Many people with Parkinson disease have symptoms related to their muscles (movement symptoms). These include stiffness, slowness in carrying out movements, and trouble with walking, getting up from a chair, or using their hands. However, people living with Parkinson disease can also have symptoms that are not related to their movement (nonmovement symptoms). These nonmovement symptoms include things like problems in thinking and memory, pain, abnormal body sensations, difficulty with emptying bowels or troubles with the bladder. Many people do not know that these other symptoms (nonmovement symptoms) may be related to Parkinson disease or to how their body responds to medications taken for Parkinson disease (levodopa, prolopa, Sinemet).

For people experiencing nonmovement symptoms that change in response to Parkinson disease medications, symptoms may only occur or may get worse when medications are either working or not working.

This questionnaire only asks about the nonmovement symptoms that come and go in response to effects of Parkinson disease medications.

For each question, if the symptom was present in the past week, you will be asked to rate how bothersome it was for you. The choices of answers are mild, moderate, or severe.

Mild. The symptom did not affect my ability to carry out normal daily tasks or social activities

Moderate. The symptom affected but did not prevent me from carrying out normal daily tasks or social activities

Severe. Symptom prevented me from carrying out normal daily tasks or social activities.

\section{NoMoFA.}

Investigator:

Subject ID:

Date Performed:

Day Month Year

(1) In the last week, did you lose your train of thought?

Yes

No

If you answered "yes", did this get better or worse after you took your levodopa?

Yes

No

Answer ONE of the following only if you answered "yes" to above: 
Was losing your train of thought related to when your levodopa WAS working?

Please rate the severity.

Mild

Moderate

Severe

\section{OR}

Was losing your train of thought related to when your levodopa WAS NOT working?

Please rate the severity.

Mild

Moderate

Severe

(2) In the last week, did you get distracted from completing a task?

Yes

No

If you answered "yes", did this get better or worse after you took your levodopa?

Yes

No

Answer ONE of the following only if you answered "yes" to above:

Was getting distracted related to when your levodopa WAS working?

Please rate the severity.

Mild

Moderate

Severe

OR

Was getting distracted related to when your levodopa WAS NOT working?

Please rate the severity.

Mild

Moderate

Severe

(3) In the last week, did you have difficulty planning or carrying out an activity (e.g., planning a party, making a grocery list, planning a menu, etc.)?

Yes

No

If you answered "yes", did this get better or worse after you took your levodopa?
Yes

No

Answer ONE of the following only if you answered "yes" to above:

Was difficulty planning or carrying out an activity related to when your levodopa WAS working?

Please rate the severity.

Mild

Moderate

Severe

OR

Was difficulty planning or carrying out an activity related to when your levodopa WAS NOT working?

Please rate the severity.

Mild

Moderate

Severe

(4) In the last week, were you disoriented (such that you did not know what day it was, or where you were, or what you were doing)?

\section{Yes \\ No}

If you answered "yes", did this get better or worse after you took your levodopa?

Yes

No

Answer ONE of the following only if you answered "yes" to above:

Was disorientation related to when your levodopa WAS working?

Please rate the severity.

Mild

Moderate

Severe

OR

Was disorientation related to when your levodopa WAS NOT working?

Please rate the severity.

Mild

Moderate

Severe

(5) In the last week, were you confused such that you had difficulty performing simple tasks (e.g., prepare a cup of tea, make a phone call)? 
Yes

No

If you answered "yes", did this get better or worse after you took your levodopa?

Yes

No

Answer ONE of the following only if you answered "yes" to above:

Was confusion related to when your levodopa WAS working?

Please rate the severity.

Mild

Moderate

Severe

OR

Was confusion related to when your levodopa WAS NOT working?

Please rate the severity.

Mild

Moderate

Severe

(6) In the last week, did you have difficulty finding the right words when speaking?

\section{Yes}

No

If you answered "yes", did this get better or worse after you took your levodopa?

Yes

No

Answer ONE of the following only if you answered "yes" to above:

Was difficulty finding the right words related to when your levodopa WAS working?

Please rate the severity.

Mild

Moderate

Severe

OR

Was difficulty finding the right words related to when your levodopa WAS NOT working?

Please rate the severity.
Mild

Moderate

Severe

(7) In the last week, were you excessively worried?

Yes

If you answered "yes", did this get better or worse after you took your levodopa?

Yes

No

Answer ONE of the following only if you answered "yes" to above:

Was feeling excessively worried related to when your levodopa WAS working?

Please rate the severity.

Mild

Moderate

Severe

\section{OR}

Was feeling excessively worried related to when your levodopa WAS NOT working?

Please rate the severity.

Mild

Moderate

Severe

(8) In the last week, did you feel scared or threatened?

Yes

No

If you answered "yes", did this get better or worse after you took your levodopa?

Yes

No

Answer ONE of the following only if you answered "yes" to above:

Was feeling scared or threatened related to when your levodopa WAS working?

Please rate the severity.

Mild

Moderate

Severe 
OR

Was feeling scared or threatened related to when your levodopa WAS NOT working?

Please rate the severity.

Mild

Moderate

Severe

(9) In the last week, did you feel restless?

Yes

No

If you answered "yes", did this get better or worse after you took your levodopa?

Yes

No

Answer ONE of the following only if you answered "yes" to above:

Was feeling restless related to when your levodopa WAS working?

Please rate the severity.

Mild

Moderate

Severe

OR

Was feeling restless related to when your levodopa WAS NOT working?

Please rate the severity.

Mild

Moderate

Severe

(10) In the last week, did you feel sad or hopeless?

Yes

No

If you answered "yes", did this get better or worse after you took your levodopa?

Yes

No

Answer ONE of the following only if you answered "yes" to above:

Was feeling sad or hopeless related to when your levodopa WAS working?

Please rate the severity.

Mild

Moderate

Severe

\section{OR}

Was feeling sad or hopeless related to when your levodopa WAS NOT working?

Please rate the severity.
Mild
Moderate
Severe

(11) In the last week, were you more likely to feel alone?

$$
\begin{aligned}
& \text { Yes } \\
& \text { No }
\end{aligned}
$$

If you answered "yes", did this get better or worse after you took your levodopa?

Yes

No

Answer ONE of the following only if you answered "yes" to above:

Was feeling isolated related to when your levodopa WAS working?

Please rate the severity.

Mild

Moderate

Severe

OR

Was feeling isolated related to when your levodopa WAS NOT working?

Please rate the severity.
Mild
Moderate
Severe

(12) In the last week, did you see things or people that were not there?

Yes

No

If you answered "yes", did this get better or worse after you took your levodopa?

Yes

No

Was seeing things or people that were not there related to when your levodopa WAS working?

Please rate the severity.

Mild

Moderate

Severe 
OR

Was seeing things or people that were not there related to when your levodopa WAS NOT working?

Please rate the severity.

\section{Mild \\ Moderate \\ Severe}

(13) In the last week, did you make poor decisions?

Yes

No

If you answered "yes", did this get better or worse after you took your levodopa?

Yes

No

Answer ONE of the following only if you answered "yes" to above:

Was making poor decisions related to when your levodopa WAS working?

Please rate the severity.

Mild

Moderate

Severe

OR

Was making poor decisions related to when your levodopa WAS NOT working?

Please rate the severity.

Mild

Moderate

Severe

(14) In the last week, were you more likely to act quickly without thinking things through?

Yes

No

If you answered "yes", did this get better or worse after you took your levodopa?

Yes

No

Answer ONE of the following only if you answered "yes" to above:

Was acting without thinking things through related to when your levodopa WAS working?

Please rate the severity.
Mild

Moderate

Severe

\section{OR}

Was acting without thinking things through related to when your levodopa WAS NOT working?

Please rate the severity.
Mild
Moderate
Severe

(15) In the last week, were you more likely to have a strong uncontrollable urge to do things (like gamble, eat too much, spend too much money or have more frequent thoughts about sexual activity)?

$$
\text { Yes }
$$

No

If you answered "yes", did this get better or worse after you took your levodopa?

Yes

No

Answer ONE of the following only if you answered "yes" to above:

Was having a strong uncontrollable urge to do things related to when your levodopa WAS working?

Please rate the severity.

Mild

Moderate

Severe

OR

Was having a strong uncontrollable urge to do things related to when your levodopa WAS NOT working?

Please rate the severity.

Mild

Moderate

Severe

(16) In the last week, did you have poor short-term memory (like putting things down and forgetting where you put them)?

Yes

No

If you answered "yes", did this get better or worse after you took your levodopa? 
Yes

No

Answer ONE of the following only if you answered "yes" to above:

Was poor short-term memory related to when your levodopa WAS working?

Please rate the severity.

Mild

Moderate

Severe

OR

Was poor short-term memory related to when your levodopa WAS NOT working?

Please rate the severity.

Mild

Moderate

Severe

(17) In the last week, did you have difficulty handling stressful situations or feel overwhelmed in stressful situations?

$$
\begin{aligned}
& \text { Yes } \\
& \text { No }
\end{aligned}
$$

If you answered "yes", did this get better or worse after you took your levodopa?

$$
\text { Yes }
$$$$
\text { No }
$$

Answer ONE of the following only if you answered "yes" to above:

Was difficulty handling stressful situations related to when your levodopa WAS working?

Please rate the severity.

\section{Mild}

Moderate

Severe

OR

Was difficulty handling stressful situations related

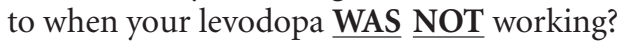

Please rate the severity.

Mild

Moderate

Severe

(18) In the last week, did you lose interest in activities that you previously enjoyed?
Yes

No

If you answered "yes”, did this get better or worse after you took your levodopa?

$$
\text { Yes }
$$

No

Answer ONE of the following only if you answered "yes" to above:

Was losing interest in activities that you previously enjoyed related to when your levodopa WAS working?

Please rate the severity.

\section{Mild}

Moderate

Severe

OR

Was losing interest in activities that you previously enjoyed related to when your levodopa WAS NOT working?

Please rate the severity.

$$
\begin{aligned}
& \text { Mild } \\
& \text { Moderate }
\end{aligned}
$$

Severe

(19) In the last week, did you feel sluggish or have low energy levels?

\section{Yes}

No

If you answered "yes", did this get better or worse after you took your levodopa?

$$
\text { Yes }
$$

No

Answer ONE of the following only if you answered "yes" to above:

Was feeling sluggish or having low energylevels related to when your levodopa WAS working?

Please rate the severity.

Mild

Moderate

Severe

\section{OR}

Was feeling sluggish or having low energylevels related to when your levodopa WAS NOT working?

Please rate the severity. 
Mild

Moderate

Severe

(20) In the last week, did you feel excessively sleepy during the day?

$$
\text { Yes }
$$$$
\text { No }
$$

If you answered "yes", did this get better or worse after you took your levodopa?

Yes

No

Answer ONE of the following only if you answered "yes" to above:

Was feeling excessively sleepy during the day related to when your levodopa WAS working?

Please rate the severity.

\section{Mild}

Moderate

Severe

\section{OR}

Was feeling excessively sleepy during the day related to when your levodopa WAS NOT working?

Please rate the severity.

\section{Mild}

Moderate

Severe

(21) In the last week, did you have painful sensations in your body (e.g., aching, tightness, burning, or sharp, dull or throbbing pain)?

$$
\text { Yes }
$$$$
\text { No }
$$

If you answered "yes", did this get better or worse after you took your levodopa?

Yes

No

Answer ONE of the following only if you answered "yes" to above:

Was having painful sensations in your body related to when your levodopa WAS working?

Please rate the severity.

\section{Mild}

Moderate

Severe

\section{OR}

Was having painful sensations in your body related to when your levodopa WAS NOT working?

Please rate the severity.

$$
\begin{aligned}
& \text { Mild } \\
& \text { Moderate } \\
& \text { Severe }
\end{aligned}
$$

(22) In the last week, did you have strange sensations in your body

$$
\text { Yes }
$$

No

If you answered "yes", did this get better or worse after you took your levodopa?

$$
\text { Yes }
$$

No

Answer ONE of the following only if you answered "yes" to above:

Was having strange sensations in your body related to when your levodopa WAS working?

Please rate the severity.

Mild

Moderate

Severe

\section{OR}

Was having strange sensations in your body related to when your levodopa WAS NOT working?

Please rate the severity.

$$
\begin{aligned}
& \text { Mild } \\
& \text { Moderate } \\
& \text { Severe }
\end{aligned}
$$

(23) In the last week, did you feel short of breath?

$$
\text { Yes }
$$

No

If you answered "yes", did this get better or worse after you took your levodopa?

$$
\begin{aligned}
& \text { Yes } \\
& \text { No }
\end{aligned}
$$

Answer ONE of the following only if you answered "yes" to above:

Was feeling short of breath related to when your levodopa WAS working?

Please rate the severity. 
Mild

Moderate

Severe

OR

Was feeling short of breath related to when your levodopa WAS NOT working?

Please rate the severity.

\section{Mild}

Moderate

Severe

(24) In the last week, did you have problems with vision (such as seeing double or things appearing blurry)?

Yes

No

If you answered "yes", did this get better or worse after you took your levodopa?

Yes

No

Answer ONE of the following only if you answered "yes" to above:

Were problems with vision related to when your levodopa WAS working?

Please rate the severity.

Mild

Moderate

Severe

OR

Were problems with vision related to when your levodopa WAS NOT working?

Please rate the severity.

\section{Mild}

Moderate

Severe

(25) In the last week, did you have an increase in sweating (such that your clothes were damp or stained from sweat more than in the past)?

$$
\text { Yes }
$$$$
\text { No }
$$

If you answered "yes", did this get better or worse after you took your levodopa?

Answer ONE of the following only if you answered "yes" to above:
Was the change in sweating related to when your levodopa WAS working?

Please rate the severity.

Mild

Moderate

Severe

OR

Was the change in sweating related to when your levodopa WAS NOT working?

Please rate the severity.

Mild

Moderate

Severe

(26) In the last week, did you feel that your heart was racing, had skipped a beat, or was pounding?

Yes

No

If you answered "yes", did this get better or worse after you took your levodopa?

Yes

No

Answer ONE of the following only if you answered "yes" to above:

Were heart racing, skipping a beat, or pounding related to when your levodopa WAS working?

Please rate the severity.

Mild

Moderate

Severe

OR

Were heart racing, skipping a beat, or pounding related to when your levodopa WAS NOT working?

Please rate the severity.

Mild

Moderate

Severe

(27) In the last week, did you urinate more frequently or had to get to the bathroom urgently?

Yes

No

If you answered "yes", did this get better or worse after you took your levodopa? 
Yes

No

Answer ONE of the following only if you answered "yes" to above:

Were urinating more frequently or having to get to the bathroom urgently related to when your levodopa WAS working?

Please rate the severity.

Mild

Moderate

Severe

OR

Were urinating more frequently or having to get to the bathroom urgently related to when your levodopa WAS NOT working?

Please rate the severity.

Mild

Moderate

Severe

(28) In the last week, did you have difficulty having a bowel movement?

Yes

No

If you answered "yes", did this get better or worse after you took your levodopa?

Yes

No

Answer ONE of the following only if you answered "yes" to above:

Was difficulty having a bowel movement related to when your levodopa WAS working?

Please rate the severity.

Mild

Moderate

Severe

OR

Was difficulty having a bowel movement related to when your levodopa WAS NOT working?

Please rate the severity.

Mild

Moderate

Severe
Do you have any other nonmovement symptoms that come and go depending on when you took your Parkinson medications that you feel are important and that were NOT mentioned in this questionnaire? If so, please describe:

Please only respond to this section if this is the SECOND TIME responding to the questionnaire:

Today, are your PD movement symptoms $\square$ better, worse, or $\square$ the same as when you answered the first questionnaire?

Today, are your PD nonmovement symptoms better, $\square$ worse, or $\square$ the same as when you answered the first questionnaire?

Are you taking the same PD medication today compared to last week? If not, please list the changes.

\section{Acknowledgments}

The authors thank Dr. A. Siderowf for facilitation of data acquisition at Pennsylvania Hospital. They also wish to thank the clinician expert panel members: Drs. Anderson, Miyasaki, Samuel, Siderowf, Shulman, Stacy, Voon, and Weiner. This study was supported by a competitive pilot project grant from the Philadelphia VA Medical Center.

\section{References}

[1] K. R. Chaudhuri, D. G. Healy, and A. H. V. Schapira, "Nonmotor symptoms of Parkinson's disease: diagnosis and management," The Lancet Neurology, vol. 5, no. 3, pp. 235-245, 2006.

[2] K. R. Chaudhuri, L. Yates, and P. Martinez-Martin, "The nonmotor symptom complex of Parkinson's disease: a comprehensive assessment is essential," Current Neurology and Neuroscience Reports, vol. 5, no. 4, pp. 275-283, 2005.

[3] O. Hornykiewicz, "Biochemical aspects of Parkinson's disease,” Neurology, vol. 51, no. 2a supplement 2, pp. S2-S9, 1998.

[4] K. Widnell, "Pathophysiology of motor fluctuations in Parkinson's disease," Movement Disorders, vol. 20, supplement 11, pp. S17-S22, 2005.

[5] W. A. Kukull, E. B. Larson, L. Teri, J. Bowen, W. McCormick, and M. L. Pfanschmidt, "The mini-mental state examination score and the clinical diagnosis of dementia," Journal of Clinical Epidemiology, vol. 47, no. 9, pp. 1061-1067, 1994.

[6] C. Moore, Nominal Group Technique in Group Techniques for Idea Building, Applied Social Research Methods Series, Sage, Thousand Oaks, Calif, USA, 2nd edition, 1994.

[7] J. M. Miyasaki, K. Shannon, V. Voon et al., "Practice parameter: evaluation and treatment of depression, psychosis, and dementia in Parkinson disease (an evidence-based review): report of the Quality Standards Subcommittee of the American Academy of Neurology," Neurology, vol. 66, no. 7, pp. 9961002, 2006.

[8] M. Stacy and R. Hauser, "Development of a patient questionnaire to facilitate recognition of motor and non-motor wearing-off in Parkinson's disease," Journal of Neural Transmission, vol. 114, no. 2, pp. 211-217, 2007.

[9] T. Witjas, E. Kaphan, J. P. Azulay et al., "Nonmotor fluctuations in Parkinson's disease: frequent and disabling," Neurology, vol. 59, no. 3, pp. 408-413, 2002. 
[10] L. M. Shulman, R. L. Taback, A. A. Rabinstein, and W. J. Weiner, "Non-recognition of depression and other non-motor symptoms in Parkinson's disease," Parkinsonism and Related Disorders, vol. 8, no. 3, pp. 193-197, 2002.

[11] C. G. Goetz, “The Unified Parkinson's Disease Rating Scale (UPDRS): status and recommendations," Movement Disorders, vol. 18, no. 7, pp. 738-750, 2003.

[12] K. R. Chaudhuri, P. Martinez-Martin, A. H. V. Schapira et al., "International multicenter pilot study of the first comprehensive self-completed nonmotor symptoms questionnaire for Parkinson's disease: the NMSQuest study," Movement Disorders, vol. 21, no. 7, pp. 916-923, 2006. 


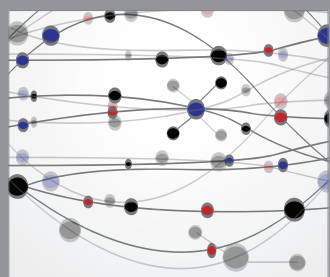

The Scientific World Journal
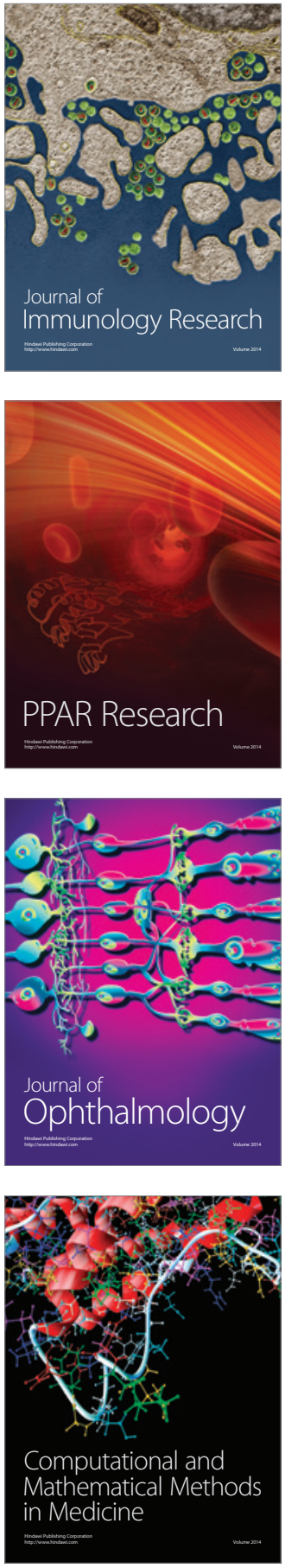

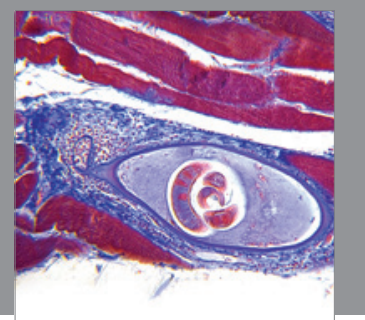

Gastroenterology

Research and Practice
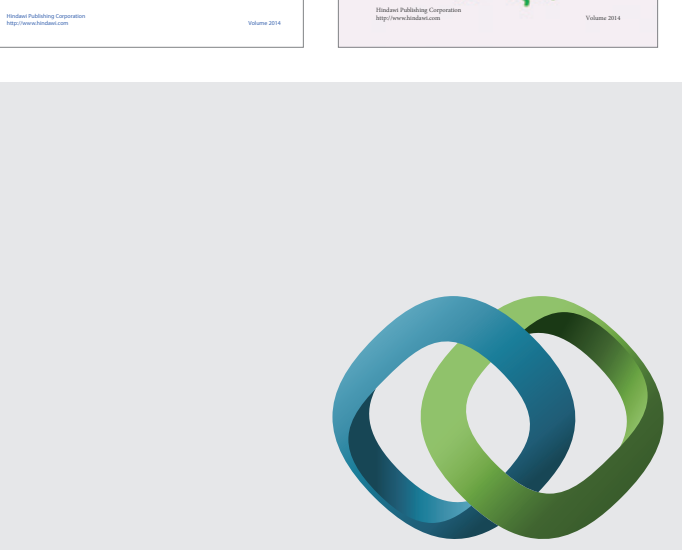

\section{Hindawi}

Submit your manuscripts at

http://www.hindawi.com
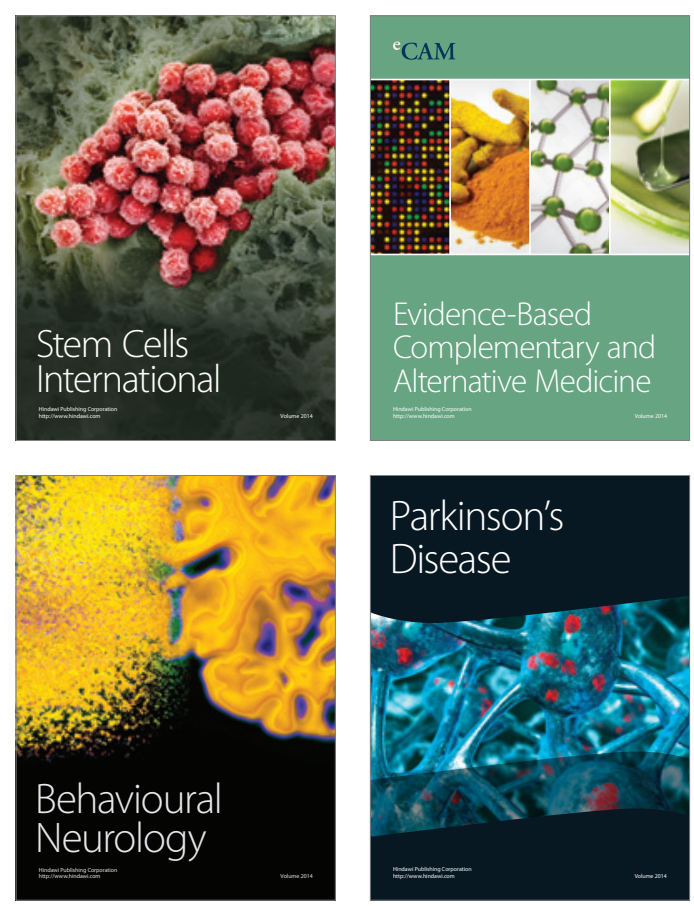

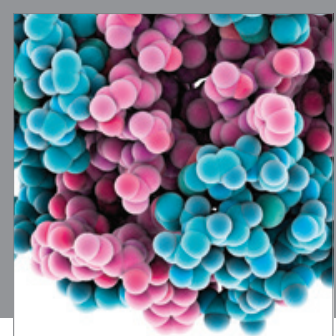

Journal of
Diabetes Research

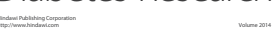

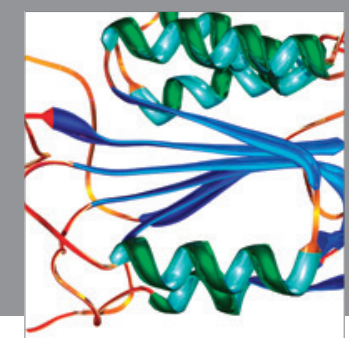

Disease Markers
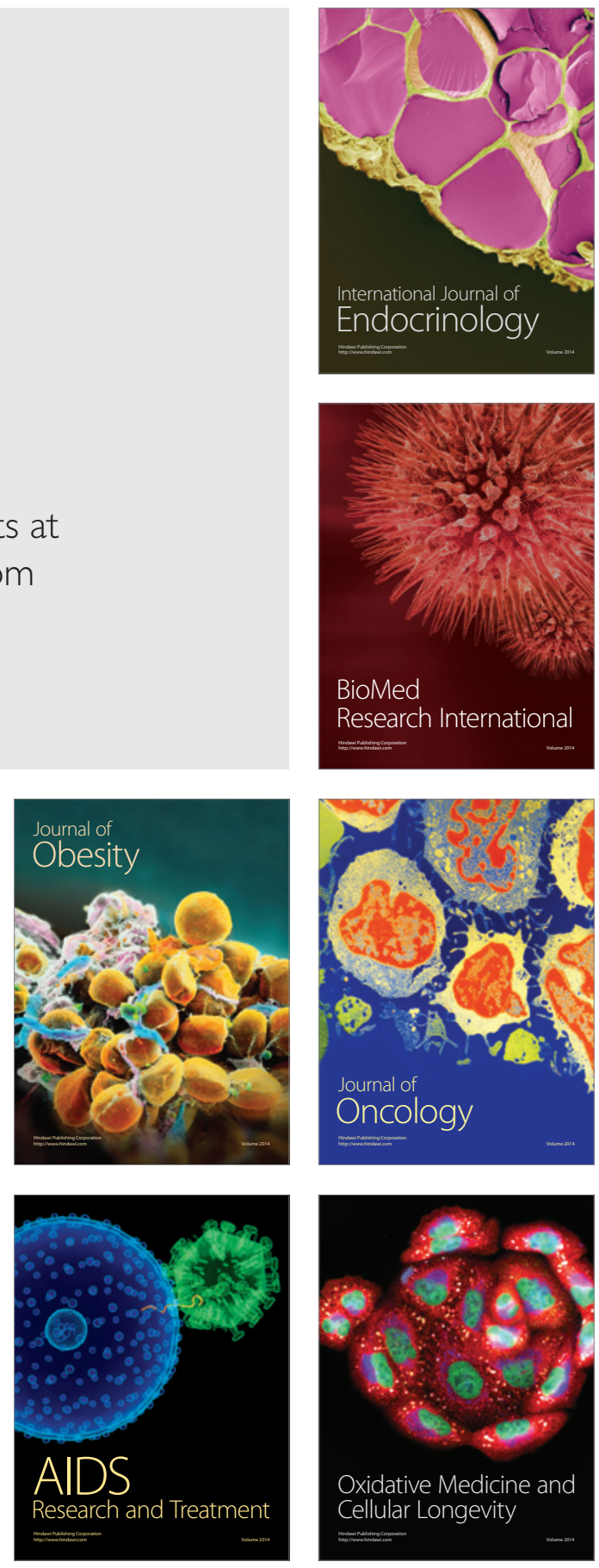